\title{
FIBROUS DYSPLASIA OF BONES.
}

\section{Orthopaedics}

\section{Darrsana}

Dr.

Venkatachalam.

$\mathbf{K}^{*}$

\section{Dr. Ampalaya} Manu R

\section{Dr. Haemanath P}

CRRI -Intern,Chettinad Hospital And Research Institute, Chettinad Academy Of Research And Education, Kelambakkam, Chengalpattu District, Pin - 603103.

Prof.And HOD, Department Of Orthopaedics, Medical Superintendent, Chettinad Hospital And Research Institute, Chettinad Academy Of Research And Education, Kelambakkam, Chengalpattu District, Pin - 603103. *CORRESPONDINGAUTHOR

MS (Ortho) PG, Department Of Orthopaedics, Chettinad Hospital And Research Institute, Chettinad Academy Of Research And Education, Kelambakkam, Chengalpattu District, Pin - 603103.

Senior Resident, Chettinad Hospital And Research Institute, Chettinad Academy Of Research And Education, Kelambakkam, Chengalpattu District, Pin - 603103.

\section{ABSTRACT}

Fibrous dysplasia is an ailment, that can include, one or a few bones and is portrayed by bone deformations, pain and fractures, arising due to bone weakening. A few patients can also present with endocrine dysfunction ( precocious puberty) and cutaneous cafe' au lait spots."

Determination depends on X-ray assessment. Anticipation is to be surveyed with X-rays and markers of bone remodelling. A few newer comprehensions, of the patho - physiology have been made in the past I0 years. It is presently perceived that fibrous dysplasia is brought about, by a physical initiating transformation of the Gs alpha subunits, of the protein G, bringing about an expanded cAMP fixation and in this way, brings about anomalies of osteoblasts separation and then subsequently these osteoblasts, creates abnormal bone. There is also an expansion in Interleukin-6 initiated osteoclastic bone resorption, which forms the basis of reasoning, for treating these patients with bisphosphonates.

In the previous 10 years, the bisphosphonate pamidronate has been utilized by imbuement for fibrous dysplasia (two courses for each year), with great outcomes regarding pain and in about half of patients, the topping off of osteolytic sores. ${ }^{[2}$

\section{KEYWORDS}

Fibrous dysplasia, Cafe' au lait spots, Mc Cune Albright syndrome.

\section{INTRODUCTION:}

Fibrous dysplasia is a bone disorder in which scar-like (fibrous) tissue develops, in place of a normal bone. This irregular tissue can weaken the affected bone and causes at times fractures. The reason for the fibrous dysplasia, is obscure. Fibrous dysplasia is generally found in the proximal femur, tibia, humerus, ribs and cranio-facial bones, in diminishing rates of occurence. Generally, presents as monostotic or polyostotic. The polyostotic form is generally more severe and is diagnosed earlier. This disease has no sexual preponderance. Cases are usually diagnosed within the first three decades of life. ${ }^{[3]}$

It is usually asymptomatic, though pain and swelling may accompany the lesion. Polyostotic fibrous dysplasia is usually associated with other disorders.

\section{Clinical symptoms: \\ PAIN: \\ Pain is only sometimes of articular starting point, aside from when secondary osteoarthritis. Agony is frequently the result of a impending crack, following a minor trauma. The intensity of pain is variable and is often aggravated by palpation. ${ }^{[4]}$}

\section{BONE DEFORMITIES:}

The shape of all long bones can be attended, giving a Shepherd's Crook distortion of the tibia or femur. ${ }^{[4]}$ The forearm or the humerus can likewise be affected. When the cranio-facial bones are involved, disfigurements can result in exophthalmy, irregularities of tooth advancement and leontiasis ostea. ${ }^{[5]}$

\section{FRACTURES :}

A pathological fracture is the regular method of finding fibrous dysplasia. $^{[3,4]}$

\section{Diagnosis :}

Diagnosis is based on clinical, radiological and pathological findings. Clinical symptoms are non-specific and diagnosis should be considered in patients with radiological features resembling those of fibrous dysplasia. In polyostotic fibrous dysplasia or in MAS, radiological findings are often sufficient, to provide for the probability of a diagnosis and a bone biopsy is generally unnecessary. In contrast, many diagnoses are possible in the monostotic fibrous dysplasia variant and a bone biopsy may be useful so one needs to confirm, both the diagnosis of fibrous dysplasia and that, the lesion is nonmalignant. The bone biopsy is by itself, likely to resut in a fracture, which can then be difficult to heal. It should therefore be avoided, whenever possible, provided the radiological appearance allows for a differential diagnosis, to be ruled out. Bone biopsy is usually performed in children during surgical treatment of first fracture. Common differential diagnoses are Paget's disease of bone, meningioma, angioma and osteofibrous dysplasia. In Paget's disease, dedifferentiation of cortices is generally observed on X-ray and there are no cystic cavities within bone. In FD, cortical bone is skinned by cancellous fibrous processes. Diagnosis of fibrous dysplasia is also to be considered in patient with numerous characteristic cafe' au lait sports with or without precocious puberty.

\section{Radiological features:}

Radiographically, fibrous dysplasia appears as a well circumscribed lesion in a long bone with a ground glass or hazy appearance of the matrix. ${ }^{[3]}$ There is a narrow zone of transition and no periosteal reaction or soft tissue mass. The lesions are usually present in metaphysis or diaphysis with intermittent focal thinning of the overlying cortex due to scalloping from within. Radiological appearance vary from cystic, pagetoid, dense and sclerotic. Recurrent fractures through trauma in the proximal femur may lead to the appearance of shepherd's crook deformity. MRI is also helpful in assessing the extent of the lesion and recognizing possible pathological fractures and also sarcomatous change within the lesion. The lesions shows heterogeneous intermediate signal on $\mathrm{T} 1 \mathrm{~W}$ images and heterogeneously low signal on $\mathrm{T} 2 \mathrm{~W}$ images but may have regions of higher signal. Post contrast T1W images show heterogeneous contrast enhancement. Bone scans demonstrate increased tracer uptake on Tc99 bone scans. Spinal lesions are very rare and take many radiological forms, such as a lytic or pseudo-angiomatous appearance. In these cases, it is generally impossible to confirm the diagnosis only by imaging so a bone biopsy is required. 


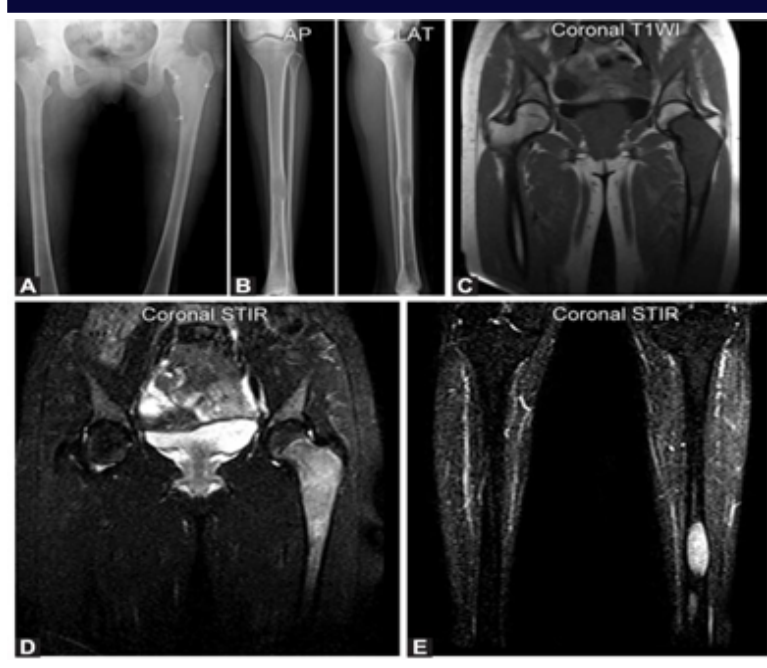

Fig 1(A to E) : Fifteen year old female patient presented with complaints of left leg pain since seven months. X-ray of both hips and thigh AP (A) view shows expansile trabeculated lytic lesion in left femoral meta-diaphysis causing expansion of medullary cavity with thinning of cortex. X-ray left leg including knee joint, AP and lateral view (B) shows expansile lytic lesion seen in distal third shaft of tibia causing expansion of medullary cavity and thinning of cortex. T1W coronal images shows hypo-intense lesion in meta- diaphyseal region of left femur causing expansion of medullary cavity and thinning of cortex. Coronal STIR image (D) shows the lesion as hyper-intense. STIR coronal image of left thigh shows hyper-intense lesion in distal third of left tibia causing expansion of medullary cavity and thinning of cortices. These findings suggest diagnosis of polyostotic fibrous dysplasia.
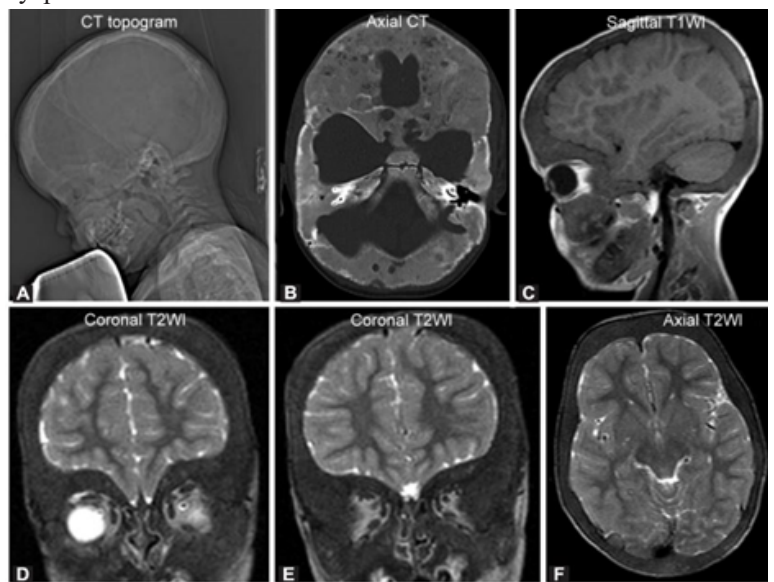

Fig 2 (A to F) : CT images showing (A) diffuse thickening of calvarium. Axial CT (B) may reveal diffuse thickening of calvarium, skull base with ground glass appearance. Sagittal T1W1 (C), sagittal coronal and axial T2-weighted images (D to F) shows diffuse thickening of calvarium, skull base and maxilla facial bones.

\section{Histological findings:}

The most prominent histological characteristic of FD is fibrous tissue made up of immature mesenchymal cells, expanding from medullary cavity to cortical bone. Long, spindle-shaped fibroblast-like cells are arranged in parallel arrays or whirls. They are embedded in a matrix of parallel collagen fibrils. These spindle- shaped cells are abnormally differentiated so an osteoblastic phenotype as they express alkaline phosphatase. Spicules of woven bone are located in fibrous tissue. These osteoblasts, involved in the deposition of lesional bone, that is, produce a bone matrix which is rich in certain anti-adhesion molecules and decreased pro-adhesive molecules like osteopontin and bone sialoprosein. Islands of hyaline cartilage can sometimes be noted embedded in fibrous tissue. ${ }^{[6]}$

Recently, 3 primary, but distinct, histological patterns have been described in Chinese literature, sclerotic/Pagetoid and sclerotic/ hypercellular types -that seem to be characteristically associated with axial or appendicular skeleton, cranial bones and gnathic bones respectively. Outer cortical bone is often invaded by FD as a consequence of action of surrounding osteoclasts, that results in cortical skinning (Figure 3)

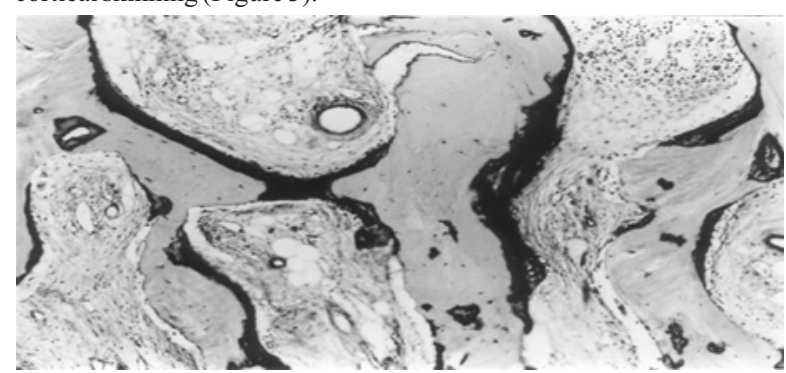

Fig 3: Histological aspect of fibrous dysplasia (Goldner staining). Note the fibrous tissue in bone marrow. Signs of osteomalacia are also present.

\section{McCune-Albright syndrome and other associations:}

McCune-Albright syndrome (MAS) was first described as a triad defined by co- occurrence of precocious puberty, polyostotic forms of fibrous dysplasia and areas of brown hyper pigmentation of skin

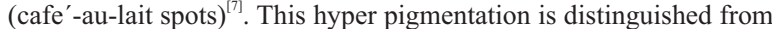
that of neuro fibromatosis by shape of its borders, which are more irregular and similar to the coast of Maine, in contrast to those of neuro fibromatosis, which are smoother, being more similar to coast of California (Figure 4)

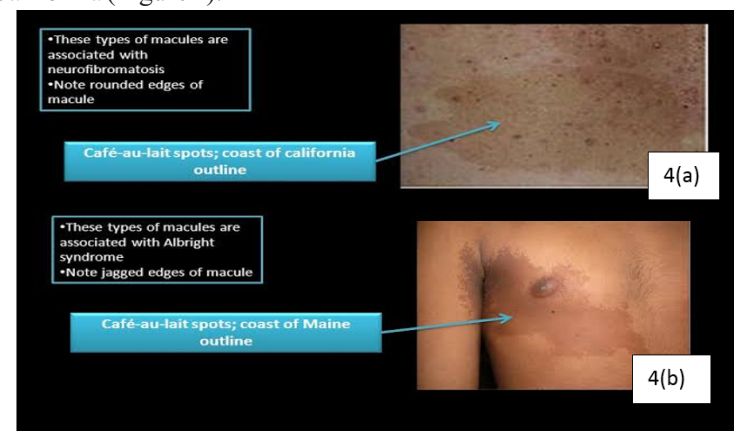

Fig 4 (a): Cafe' -au-lait spots seen in cases of neurofibromatosis.

4(b) : Cafe' -au-lait spots seen in cases of McCune Albright Syndrome.

These cafe' -au-lait spots are usually on same side of body as bone involvement, often being arranged in a segmental pattern that follows development. Multiple endocrine abnormalities may co-exist, for example thyroid nodules and hyperthyroidism, adrenal hyperplasia and hypercortisolism, pituitary tumours with acromegaly or hyper prolactinemia and hypophosphataemic rickets and osteomalacia resulting from a reduced resorption of phosphate from renal tubules. Non-endocrine abnormalities are occasionally present, affecting liver, spleen, bone marrow and brain. However, vast majority of patients present with only two of features of triad and are considered to have MAS.

The co-existence of fibrous dysplasia and the intramuscular myxomas may be seen in some patients (Mazabraud's syndrome), particularly those with polyostotic fibrous dysplasia and MAS. These myxomas are often large and multiple.

\section{CONCLUSION:}

The FD/MAS persistent population is assorted, with interesting needs that range different clinical and dental strengths. Conservative management is the mainstay of treatment in asymptomatic cases.It is pivotal for FD/MAS patients to be treated by a group, including doctors, specialists and dental authorities. It is critical to have the option to perceive the trademark indications of FD/MAS, not withstanding realizing how to allude the patient for a proper workup. It is additionally basic to comprehend the ramifications of MASrelated endocrinopathies, for example, excessive growth hormone and how these conditions can advise treatment choices.

\section{REFERENCES}

Lichtenstein L \& Jae A HL. Fibrous dysplasia of bone. Archives of Pathology 1942; 33 $777 \pm 816$.

Coley B. Neoplasms of Bone and Related Conditions, vol. 1. New York: Paul Hocher, 
3. Henry A. Monostotic ${ }^{\circledR}$ brous dysplasia. Journal of Bone and Joint Surgery 1969; 51B: $300 \pm 306$.

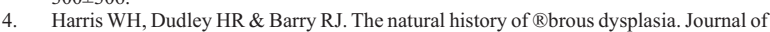
Bone and JointSurgery 1962;44A: $207 \pm 233$.

5. Mohammadi-Araghi H \& Haery C. Fibro-osseous lesions of craniofacial bones. Radiologic Clinics of NorthAmerica 1993; 31: 121 134 .

6. Lucas E, Sundaram M \& Boccini T. Radiological case study: polyostotic ®brous dysplasia. Orthopedics 1995;18:311 \pm 313 .

7. McCune DJ. Osteitis $\mathbb{R}$ brosa cystica: the case of a nine year old girl who also exhibits precocious puberty,multiple pigmentation of the skin and hyperthyroidism. American Journal of Diseases of Children 1936; 52:743 \pm 74 . 